\title{
Genericity Versus Inheritance
}

\author{
Bertrand Meyer \\ Interactive Software Engineering, Inc., 270 Storke Road, Suite \#7, Goleta CA 93117
}

\begin{abstract}
Genericity, as in Ada or $M L$, and inheritance, as in object-oriented languages, are two alternative techniques for ensuring better extendibility, reusability, and compatibility of software components. This article is a comparative analysis of these two methods. It studies their similarities and differences and assesses to what extent each may be simulated in a language offering only the other. It shows what features are needed to successfully combine the two approaches in an object-oriented language that also features strong type checking. The discussion introduces the principal aspects of the language Eiffel ${ }^{\text {TMI }}$ whose design, resulting in part from this study, includes multiple inheritance and a limited form of genericity under full static typing.
\end{abstract}

\section{OVERVIEW}

Despite its name, today's software is usually not soft enough: adapting it to new uses turns out in most cases to be a harder endeavor than should be. It is thus essential to find ways of enhancing such software quality factors as extendibility (the ease

This article is a revised version of a paper presented at OOPSLA (First ACM Symposium on Object-Oriented Programming Systems, Languages and Applications, Portland, Oregon, Sept. 29-Oct. 2, 1986), Ed. Norman Meyrowitz, published as Sigplan Notices, 21, 11, pp. 391-405.

Trademarks: Unix (AT\&T); Ada (U.S. DoD); Eiffel (Interactive Software Engineering). with which a software system may be changed to account for modifications of its requirements), reusability (the ability of a system to be reused, in whole or in parts, for the construction of new systems), and compatibility (the ease of combining a system with others).

Good answers to these issues are not purely technical, but must include economical and managerial components as well; and their technical aspects extend beyond programming language features, to such obviously relevant concerns as specification and design techniques. It would be wrong, however, to underestimate the technical aspects and, among these, the role played by proper programming language features: any acceptable solution must in the end be expressible in terms of programs, and programming languages fundamentally shape the software designers' way of thinking.

This article is a comparative analysis of two classes of programming language features for enhancing extendibility, reusability, and compatibility. It assesses their respective strengths and weaknesses, examines which of their components are equivalent and which are truly different, shows how the two approaches complement each other, and explains how they have been combined in a particular programming language design.

The two approaches studied are genericity and inheritance; both address the above issues by allowing the definition of flexible software elements amenable to extension, reuse, and combination. The first is a technique for defining elements that have more than one interpretation, depending on parameters representing types; the second makes it possible to 


\section{Genericity versus Inheritance}

define elements as extensions or restrictions of previously defined ones.

Both methods apply some form of polymorphism, a notion that may be defined as the ability to define program entities that may take more than one form. A simple form of polymorphism, used in both cases, is overloading, the ability to attach more than one meaning to the same name, ambiguities being resolved by examining the context of each occurrence of the name, either at compile time (for statically typed languages) or at run time.

Although the two approaches may be applied outside the strict realm of programming, for example to specification or design languages, we shall confine our study to programming languages. In this field, genericity is most notably present in Ada; inheritance is a feature of object-oriented languages and was introduced by Simula 67 .

Ada and object-oriented languages have until now aroused interest in rather different communities and it is not surprising that no comparative analysis seems to have been published. (The only related work that we know of is the as yet unpublished, more theory-oriented article by Cardelli and Wegner [6], of which we became aware as this paper was going to press.) However, we feel that beyond "cultural" differences the real goals pursued are the same, so that it is fruitful to perform an in-depth comparison of the technical solutions obtained on both sides.

\section{GENERICITY}

Genericity as offered by Ada is present in few other programming languages (examples include CLU [10] and LPG [2]), but is offered by several formal specification languages, such as Z [1], Clear [5], OBJ2 [9], and LM [15]. A variant of this approach was developed in connection with the language ML [16, 7] and has been integrated into a number of functional languages.

We shall concentrate on the Ada form, restricting ourselves to type genericity, that is to say the ability to parameterize a software element (in Ada, a package or subprogram) by one or more types. Generic parameters have other, less interesting uses in Ada, such as parameterized dimensions for arrays.

We shall distinguish between unconstrained genericity, whereby no specific requirement is imposed on generic parameters, and constrained genericity, whereby a certain structure is required.

\section{Unconstrained Genericity}

In its simplest form, unconstrained genericity may be seen as a technique to bypass the unneces sary requirements imposed by static type checking

Consider the example of a simple procedure for exchanging the values of two variables. In a lan. guage which is not statically typed, such as Lisp, we would write something like the following, syntactic differences notwithstanding:

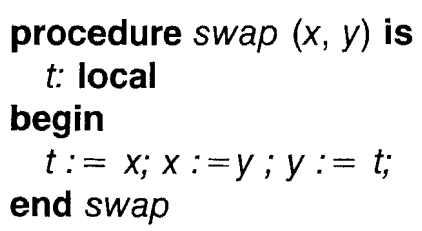

The type of the elements to be swapped and of the local variable $t$ does not have to be specified. However, this may be too much freedom since a call of the form $\operatorname{swap}(a, b)$, where $a$ is, say, an integer; and $b$ a character string, will not be prohibited even though it is probably an error.

To address this issue, statically typed languages, such as Pascal, require programmers to ex. plicitly declare the types of all variables and formal parameters, and enforce a statically checkable type compatibility constraint between actual and formal parameters in calls and between source and target in assignments. In such a language, the procedure to exchange the values of two variables of type $T$ becomes:

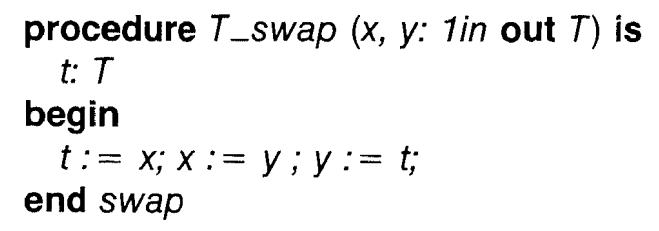

Demanding that $T$ be specified as a single type averts type incompatibility errors, but has the unpleasant consequence of requiring a new procedure declaration for each type for which a swap operation is needed; in the absence of overloading, a different name must be assigned to each such procedure, for example int_swap, str_swap and so on. Such multiple declarations lengthen and obscure programs. The example chosen is particularly bad since all the declarations will be identical except for the two occurrences of $T$.

Static typing may be considered too restrictive here: the only real requirement is that the two actual parameters passed to any call of swap should be of 


\section{Genericity versus Inheritance}

the same type; and that their type should also be applied to the declaration of the local variable $t$.

A language with genericity provides a tradeoff between too much freedom, as with untyped languages, and too much restraint, as with Pascal. In such a language, one may declare $T$ as a generic type parameter to the swap procedure. In quasi-Ada, the procedure may be declared as follows

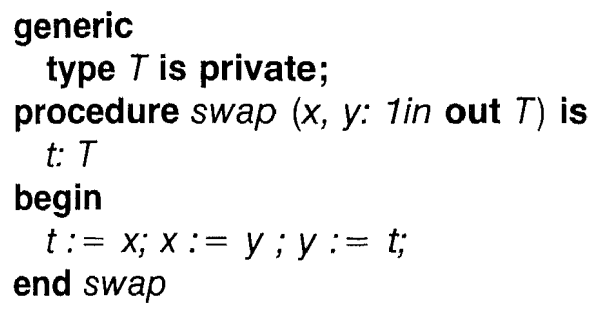

The only difference with real Ada is that we have merged together, for ease of presentation, the two parts of an Ada subprogram declaration, header and body; their separation in Ada comes from a concern for information hiding, orthogonal to this discussion.

The generic. . clause introduces type parameters. By specifying $T$ as "private", the writer of this procedure allows himself to apply to objects of type $T(x, y$, and $t)$ operations available on all types, such as assignment or comparison, and these only.

A declaration such as the above does not actually introduce a procedure but rather a procedure pattern; actual procedure instances are obtained by providing actual type parameters, as in

\section{procedure int_swap is new swap (INTEGER); procedure str_swap is new swap (STRING);}

etc. Now assuming that $i$ and $j$ are variables of type INTEGER, $s$ and $t$ of type STRING, then of the following calls

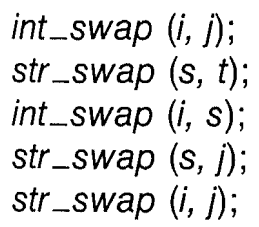

all but the first two are statically incorrect.

More interesting than parameterized subprograms are parameterized packages. Ada packages (and their equivalents in other modular languages, such as modules in Modula-2) are syntactical encapsulations of groups of related entities such as subprograms, types and variables. One of the most im- portant applications of packages, and the only one considered in this article, is data abstraction: each package contains the implementation of a type and of the operations applicable to elements of that type.

Ada packages may be declared with generic parameters. For example, the following package provides stacks of elements of an arbitrary type $T$

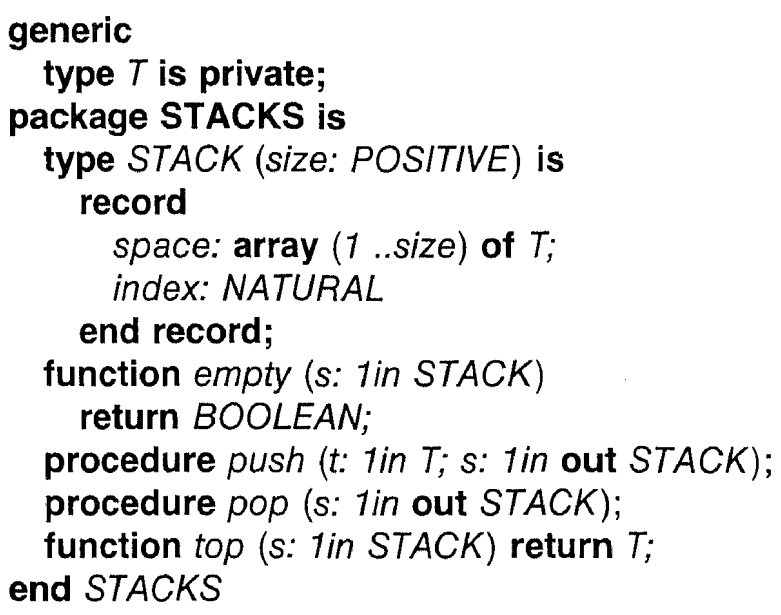

We have given only the public part ("specification") of the package; the package implementation ("body"), which describes the subprogram bodies, must be declared separately. For technical reasons having to do with the problems of Ada compilation, the implementation of the types supported by a package, such as STACK here, is given in the public part. For information hiding purposes, this implementation may be given in the private clause of the public part, a kind of purgatory between specification and body; however we do not need this feature for the present discussion.

As with generic subprograms, the above does not define a package but a package pattern; actual packages may be obtained by instantiation, as in

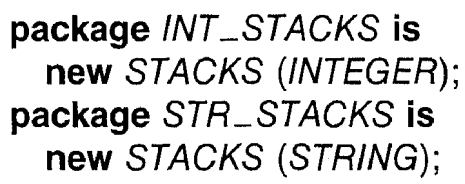

In a program unit that has access to both of these instances of STACKS, dot notation may be used to distinguish between namesake elements: for example the type "stack of integers" will be denoted by INT_STACKS.STACK, and the type "stack of strings" by STR_STACKS.STACK; the corresponding "push" procedures are INT_STACKS.push and STR_STACKS.push.

We may note again the compromise that ge- 


\section{Genericity versus Inheritance}

neric declarations achieve between typed and untyped languages. STACKS provides a pattern for the declaration of modules implementing stacks of elements of all possible types $T$, while retaining the possibility to enforce type checks: for example it will not be possible to push an integer onto a stack of strings.

Both examples above (swap and stack) evidence a form of genericity which we call unconstrained since there is no specific requirement on the types that may be used as actual generic parameters: one may swap the values of variables of any type and create stacks of values of any type-provided all the values in a given stack are of the same type.

Other generic definitions, however, only make sense if the actual generic parameters satisfy some conditions. We call this form constrained genericity.

\section{Constrained Genericity}

As in the unconstrained case, we consider two constrained examples, a subprogram and package.

Assume we want a generic function subprogram to compute the minimum of two values. We may use the pattern of swap:

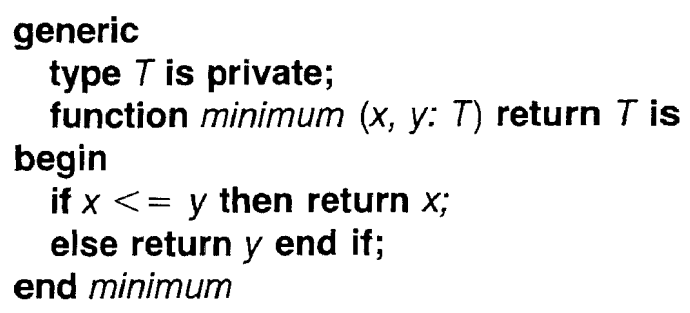

However, such a function declaration is not always meaningful: it should only be instantiated for types $T$ on which a comparison operator $<=$ is defined. In an untyped language, we might defer checking of this property until run-time, but this is not acceptable in a language that enhances security through static typing. We need a way to specify that type $T$ must be equipped with the right operation.

In Ada, this will be written by treating the operator $<=$ as a generic parameter of its own. Syntactically it is a function; as a syntactic facility, it is possible to invoke such a function using the usual infix form if it is declared with a name in double quotes, here " $<=$." Again the following declaration becomes legal Ada if the public part and implementation are taken apart.

\section{generic}

type $T$ is private; with function " $<=$ " $(a, b: T)$

return $B O O L E A N$ is $\langle>$;

function minimum $(x, y: T)$ return $T$ is

begin

if $x<=y$ then return $x$;

else return $y$ end if;

end minimum

The keyword with introduces generic parameters representing subprograms, such as " $<=$ ".

We may instantiate minimum for any type, say $T 1$, such that there exists a function, say $T 1$. le, of type function $(a, b: T 1)$ return BOOLEAN:

\section{function T1_minimum is \\ new minimum $\left(T 1, T 1 \_l e\right)$;}

If, on the other hand, function T1_le is in fact called " $<=$," that is to say if its name and type match those of the corresponding formal subprogram, then it may be omitted from the list of actual parameters to the generic instance. For example, type INTEGER has a predefined " $<=$ " function with the right type, so that we can simply declare

\section{function int_minimum is new minimum (INTEGER);}

This use of default subprograms with match. ing names and types is made possible by the clause is $\langle>$ in the declaration of the formal subprogram, here " $<=$ ". Operator overloading, as permitted (and in fact encouraged) by the design of Ada, plays an essential role here: many different types may have a " $<=$ " function.

This discussion of constrained genericity for subprograms readily transposes to packages. Assume we need a generic package for handling matrices of objects of any type $T$, with matrix sum and product as basic operations. Such a definition only makes sense if type $T$ has a sum and a product of its own, and each of these operations has a zero element; these features of $T$ will be needed in the implementation of matrix sum and product. The public part of the package may be written as follows

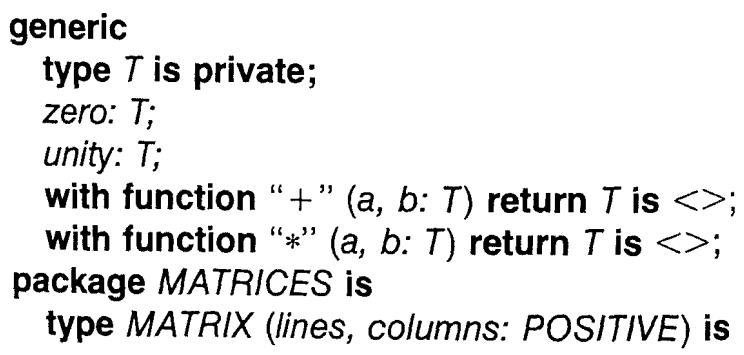




\section{Genericity versus Inheritance}

array (1 ..lines, 1 ..columns) of $T$;

function "+" ( $m 1$, m2: MATRIX)

return MATRIX;

function "*" (m1, m2: MATRIX)

return MATRIX;

end MATRICES;

Typical instances of the package are

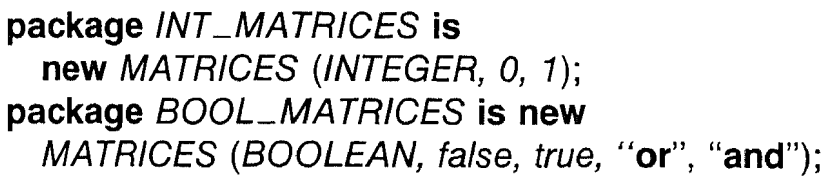

Again, actual parameters corresponding to formal generic subprograms (here " +" and " **) may be omitted for type INTEGER, which has matching operations; but they must be included for $B O O L$ $E A N$. (It is convenient to declare such parameters last in the form list; otherwise keyword notation is required in calls which omit the corresponding actual parameters.)

It is interesting here to show how the implementation part of such a package will look. It is enough to give one of the function bodies in this package; we take matrix product as an example.

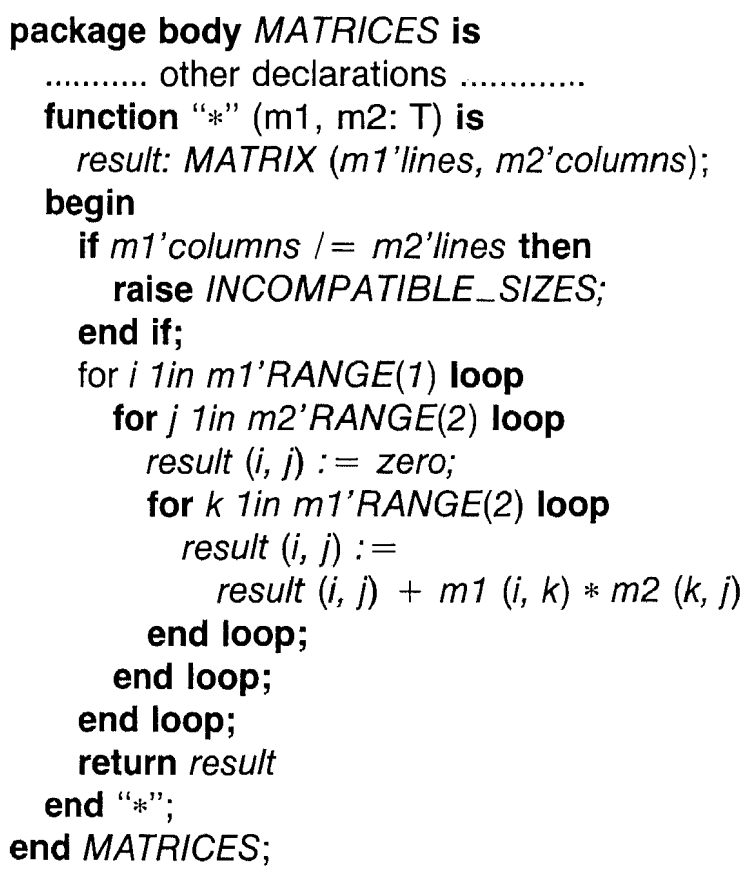

Three comments are in order for the reader not familiar with all the details of Ada:

- for a parameterized type such as MATRIX (lines, columns: POSITIVE), a variable declaration must provide actual parameters, e.g. $m m$ : $M A$ TRIX $(100,75)$; their values may then be re- trieved using the apostrophe notation as in $m m$ 'lines which in this case has value 100;

- if $a$ is an array, $a^{\prime} R A N G E$ ( $i$ ) denotes the range of values in its $i$-th dimension; for example $m I^{\prime} R A N G E(1)$ above is the same as 1 ..m1'lines;

- if requested to multiply two dimension-wise incompatible matrices, the program raises an exception; it does not execute the code that follows the raise instruction. The package should include code to handle the exception.

The minimum and matrix examples are representative of Ada techniques for constrained genericity. They also show a serious limitation of these techniques: the fact that only syntactic constraints may be expressed. All that a programmer may require is the presence of certain subprograms (" $<=$ ", "+", “*” in the examples) with given types; but the declarations are meaningless unless some semantic constraints are also satisfied. For example, minimum only makes sense if " $<=$ " is a total order relation on $T$; and the MATRICES package should not be instantiated for a type $T$ unless the operations "+" and "*" not only have the right type $(T \times T \rightarrow$ $T$ ) but also give $T$ the structure of a ring (associativity, distributivity, zero a zero element for " + " and unity for “壮, etc).

To include such formal constraints, one has to leave the realm of programming languages such as Ada for such specification languages as Clear and OBJ2 (the latter executable) or the experimental programming language LPG.

\section{Implicit Genericity}

It is important to mention a form of genericity quite different from the above Ada-style explicit parameterization: the implicit polymorphism exemplified by the work on the ML functional language $[16,7]$.

This technique is based on the remark that explicit genericity, as seen above, places an unnecessary burden on the programmer, who must give generic types even when the context provides enough information to deduce a correct typing. It may be argued, for example, that the very first version (/1/) given for procedure swap, with no type declaration, is acceptable as it stands: with adequate typing rules, a compiler has enough information to deduce that $x$, $y$, and $t$ must have the same type. Why not then let programmers omit type declarations when they are not strictly needed conceptually, and have the compiler check that all uses of an identifier are consistent?

This approach, sometimes called "unobtrusive 


\section{Genericity versus Inheritance}

type checking", attempts to reconcile the freedom of untyped languages with the security of typed ones. It has been elegantly implemented in ML and other functional languages. One may argue, of course, that some obtrusiveness may be useful; the redundancy entailed by explicit type declarations may enhance program readability. Be it as it may, the question of explicit or implicit genericity is not directly connected to the present discussion; for the purposes of comparison with inheritance, both forms of genericity are somehow equivalent.

Without committing ourselves as to which form is best, we have chosen to rely on the explicit Ada form, which, for our study, has the obvious advantage that generic parameters stand out more visibly.

\section{INHERITANCE}

The inheritance technique was introduced in 1967 by Simula $67[3,8,11]$. It has been widely imitated in other object-oriented languages.

As with genericity, we will mostly introduce this technique through examples. We shall rely on the notation of the object-oriented language Eiffel $[13,12,14] . *$ Much of the discussion would transpose to other object-oriented languages; however Eiffel's emphasis on static typing, and its design as an objectoriented language for actual software engineering applications (as opposed to, say, artificial intelligence or exploratory programming) make it particularly suitable for this discussion. Only the elements of Eiffel which are essential to this article are introduced; more details may be found in the references quoted.

The fundamental idea of inheritance is that new software elements may be defined as extensions of previously defined ones, which should not have to be modified for the occasion.

This concept blends particularly well with the object-oriented approach, in which basic software elements (modules) are implementations of abstract data types: the extensions of software elements mentioned above will then correspond to refinements of hierarchies of abstract data types.

The basic tenet of object-oriented languages may be described as the idea that modules not only contain abstract data type implementations (an ef-

*Eiffel and the associated compiler and environment are products of Interactive Software Engineering, Inc., Goleta (California). fect which may be achieved in any language offering modular features and information hiding, such as Ada or Modula-2), but are such implementations. In other words, the defining equality of object-oriented languages is

Module $\equiv$ Type

This dogmatic identification of two apparently distinct programming notions, one syntactic, the other semantic, may appear too strict and indeed has some disadvantages. But it also gives object-oriented pro. gramming languages and the associated design method a strong conceptual integrity, and provides powerful techniques for satisfying the software qual. ity requirements mentioned above.

As an example of such a module-type, called a class in Eiffel as in Simula and many other object. oriented languages, consider the following outline of an implementation of "special files" in the Unix sense, that is to say, files associated with devices

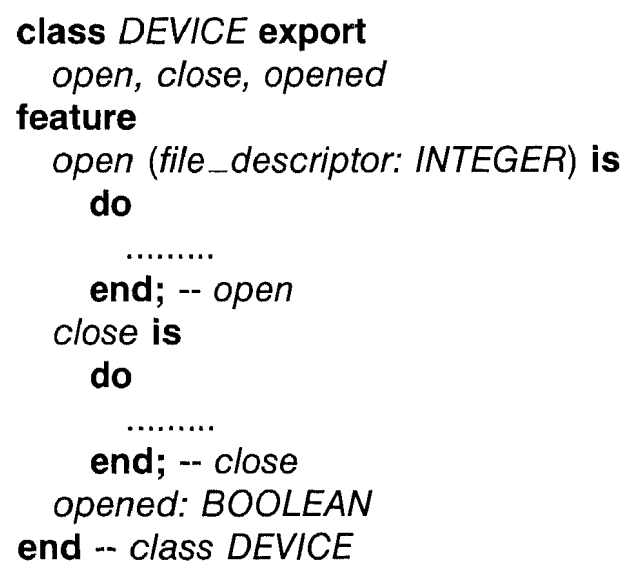

This class is the implementation of an abstract data type characterized by three "features," open, close, and opened. There are two kind of features: attributes and routines. Routines, like open and close here, are operations applicable to objects of the class; routines are further divided into procedures which, as the two shown here, perform some actions, and functions (seen in later examples), which return a value. Attribute features, like opened here, are data elements associated with each object of the type.

As a type, a class such as DEVICE may be used to declare objects; their features may then be accessed through dot notation, as in

d1: DEVICE; f1: INTEGER

d1.Create; 


\section{Genericity versus Inheritance}

d1.open (fl);

if $d 1$.opened then ....

Create is a universal procedure applicable to all classes; it allocates the necessary space for an object such as $d 1$. Further initialization, if needed, may be described in a possibly parameterized procedure declared in the class with the name Create.

Note that each routine always has, besides its normal list of arguments, a special argument, the object to which the procedure is applied ( $d 1$ in the above calls). This is characteristic of object-oriented languages: every operation is relative to a distinguished object. Within the class, unqualified feature names implicitly refer to this object; the predefined name Current may be used when an explicit reference is needed.

These comments account for the "type" aspect of a class. From the "module" standpoint, it should be noted that the class is the only program structuring facility of Eiffel; thus the above example use of DEVICE must be in some class, say $C$. A class such as $C$ which declares entities (that is to say features, routine parameters, or function results) of type $D E V I C E$ is said to be a client of DEVICE. The export clause lists the features of a class which are accessible to clients, in read-only mode for attributes and execution mode for routines (here all features shown are exported). Since information hiding is not a concern for this discussion, we shall omit export ... clauses in the sequel.

The notion of inheritance is a natural extension to this basic framework. Assume we want next to define the notion of tape device. For our purposes, a tape unit has all the properties of devices, as represented by the three features of class DEVICE, plus the ability to (say) rewind its tape. Rather than redefining a new class from scratch, we may declare class TAPE as an extension of DEVICE, as follows

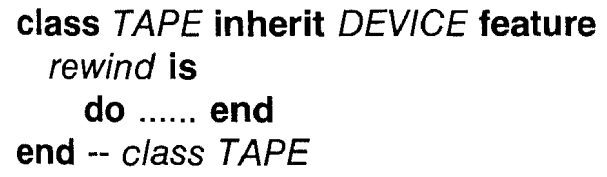

With this declaration, objects of type TAPE automatically possess (by "inheritance") all the features of DEVICE objects, plus their own (here rewind). We say that TAPE is an heir to DEVICE, which is a parent of TAPE. The "descendants" of a class are the class itself and the descendants of its heirs; the reverse notion is that of "ancestor."

A class may of course have more than one heir; for example, DEVICE could have DISK as another heir, with its own specific features (such as direct access read, etc.). In Eiffel, classes may also have more than one parent: this is known as multiple inheritance, a very powerful technique for reusability, allowing the combination of more than one previously developed environment. Eiffel also introduces the technique of "repeated inheritance," making it possible to inherit more than once from the same class.

From the module viewpoint, the ancestor relation is a program structuring mechanism; from the type viewpoint, it yields a rule on legal assignments. The rule is simple: an assignment

$x:=y$

where $x$ and $y$ are of class types, is permitted if and only if the type of $x$ is an ancestor of the type of $y$. Thus the above assignment is legal if, for example, $x$ has been declared as a device and $y$ as a tape. This may be explained by noting that the inheritance relation is really the "is-a" relation [4]: every tape is a device, but every device is not a tape.

It sometimes happens that a feature of a class should be implemented differently in some descendants of the class. For example, there could be a special "open" mechanism for tape devices. Eiffel allows such redefinitions, as follows

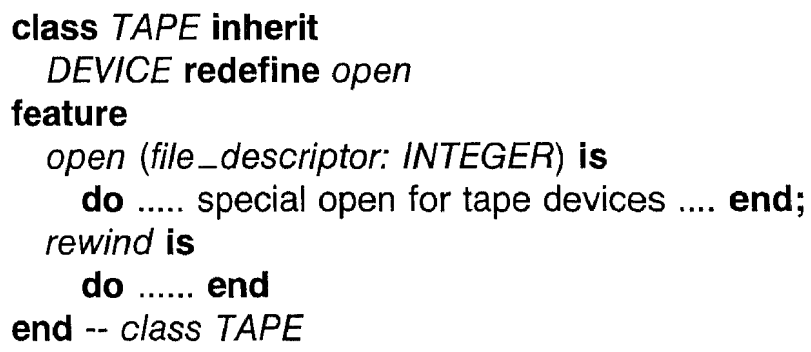

This possibility must be seen in the light of the assignment rule: if $x$ is a device, then the call

$x . o p e n(f 1)$

may be executed differently depending on the assignments performed on $x$ before the call: for example, after $x:=y$, where $y$ is a tape, the tape version should be executed. Such feature redefinitions are common in Eiffel programming, which also allows a parameterless function to be redefined as an attribute (which is useful for changing representations in program refinement).

This facility characterizes the powerful brand 


\section{Genericity versus Inheritance}

of polymorphism offered by object-oriented languages with inheritance: the same feature reference may have several interpretations depending on the actual form of the object at run-time. To achieve this effect, many object-oriented languages have renounced static type checking; Eiffel, however, is statically typed (and the binding of feature names to actual features is done statically whenever possible).

The remarkable benefits of the inheritance technique with respect to reusability, extendibility, and compatibility come from the fact that software elements such as DEVICE are both usable as they are (they may be compiled as part of an executable program) and still amenable to extensions (if used as ancestors of new classes). Thus, a compromise between usability and flexibility, fundamental for the qualities mentioned, is achieved.

One more property of Eiffel, borrowed from Simula, will be useful for the discussion below: deferred features (corresponding to Simula's "virtual procedures"). Deferred features correspond to operations that must be provided on all objects of a class, but whose implementation may only be given in particular descendants of the class.

Assume for example that, as under Unix, devices are a special kind of files; DEVICE should thus be an heir to class FILE, whose other heirs may be TEXT_FILE (itself with heirs NORMAL and DIRECTORY) and BINARY_FILE. Figure 1 shows the inheritance graph, a tree in this case.

Any file may be opened or closed; but how these operations are performed depends on whether the file is a device, a directory etc. Thus, at the FILE level we declare the corresponding procedures as deferred, giving only a header and handing over the burden of actual implementation to descendant classes

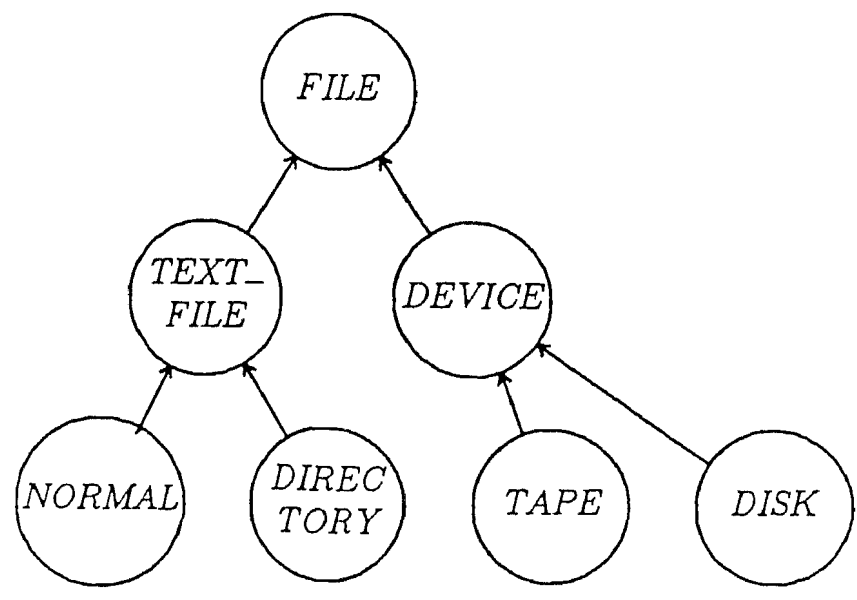

Fig. 1. Inheritance graph for files.

\author{
class FILE feature \\ open (file_descriptor: INTEGER) is deferred end; \\ close is deferred end; \\ end -- class FILE
}

Descendants of FILE should provide actual definitions of open and close. The rules of the language prohibit application of these features to objects for which they might not be defined.

An interesting application of this technique is Ada or Modula-like separation between interface and implementation of a module: although an Eiffel class is normally defined as a single piece, the effect of Ada's two-level declaration (specification and body) may be achieved by declaring a first class with deferred features only, and a second one, heir to the first, with the implementation of these features. This technique has an important advantage over its Ada equivalent: it allows different implementations of the same feature to coexist in a single software system.

\section{SIMULATING INHERITANCE WITH GENERICITY}

To compare genericity with inheritance, we shall study how, if in any way, the effect of each feature may be simulated in a language offering the other.

First consider a language such as Ada, offering genericity but not inheritance. Can it be made to achieve the effects of inheritance?

The easy part is the overloading. In a language such as Ada or Algol 68 where the same subprogram name may be reused as many times as needed provided it is applied too perands of different types, there is no difficulty in defining types such as TAPE, $D I S K$, etc., each with its own version of open, close etc.:

\section{procedure open \\ ( $p$ : 1in out TAPE; descriptor: 1in INTEGER); procedure close ( $p$ : 1 in out $D I S K)$; etc.}

Provided the subprograms are distinguished by the type of at least one operand, as is the case here, no ambiguity will arise.

Yet this solution falls short of providing true polymorphic entities as in languages with inheritance, where, as discussed above, an operation may be executed differently depending on the form of its operand at run-time (even though it is possible, at 


\section{Genericity versus Inheritance}

least in Eiffel, to check at compile time that the operation is defined in all possible cases). A typical example is the call d.close, which will be carried out differently after the assignments $d:=d i$ and $d:=$ $t a$, where $d i$ is a DISK and ta a TAPE. The Ada-like form of overloading does not provide anything like this remarkable possibility.

The only feature of Ada which could be used to emulate this property of object-oriented languages is in fact shared with Pascal and has nothing to do with overloading or genericity; it is the record with variant fields. We could for example define something like

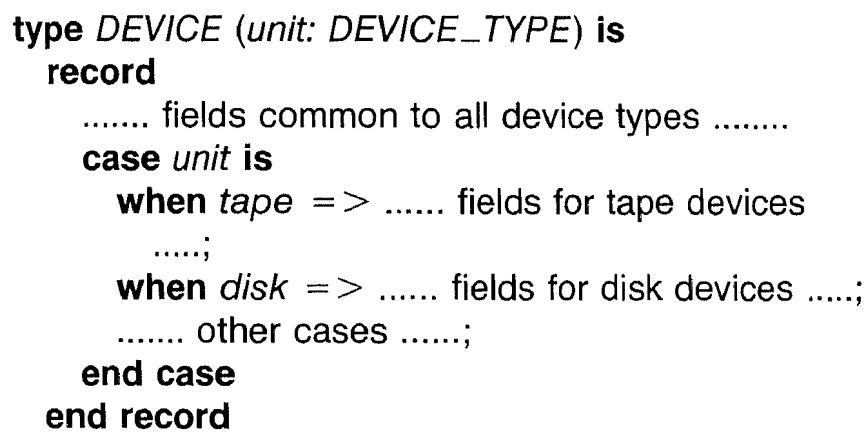

where DEVICE_TYPE is an enumeration type with elements tape, disk, etc. Then there would be a single version of each the procedures on devices (open, close etc.), each containing a case discrimination of the form

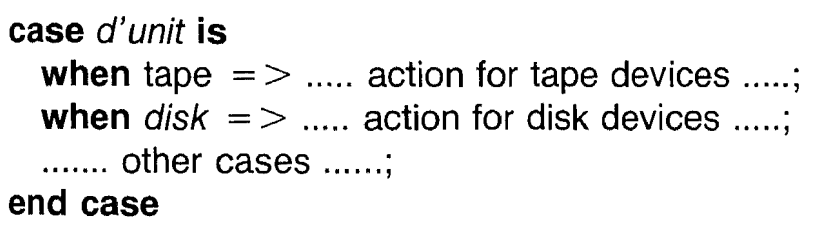

Such a solution, however, is unacceptable from a software engineering viewpoint; it runs contrary to the criteria of extendibility, reusability, and compatibility. Not only does it scatter case discriminations (here on DEVICE_TYPE) all over the program; worse yet, it closes the set of possible choices: as opposed to the Eiffel class DEVICE which can at any time be used as parent or a new class, the Ada type $D E V I C E$ has a fixed list of variants, one for each element of the enumeration type DEVICE_ TYPE. To add a new case, one must change the declaration of $D E V I C E$, invalidating any program unit that relied on it.

So the answer to the question posed at the beginning of this section-can inheritance be simulated with genericity?-is no.

\section{SIMULATING GENERICITY WITH INHERITANCE}

We now address the reverse problem: can we achieve the effect of Ada-style genericity in an object-oriented language with inheritance?

As before, we use Eiffel as our vehicle for expressing object-oriented techniques. As explained later, Eiffel does provide a generic parameter mechanism (included in the language as a result of the study reported here); but of course, since the object of this section is to analyze how one may simulate genericity with inheritance, we must temporarily refrain from using the Eiffel generic mechanism. The reader should thus be warned that the solutions presented in this section are substantially more complex than those obtainable with full Eiffel, described in the next section.

The simulation turns out to be easier, or at least less artificial, for constrained genericity-a surprising result since unconstrained genericity is conceptually simpler. Thus we begin with the constrained case.

\section{Constrained Genericity: Overview}

The idea is to associate with a constrained formal generic type parameter a class. This is a natural thing to do since a constrained generic type may be viewed, together with its constraining operations, as an abstract data type. Consider for example the generic clauses in our two constrained examples, minimum and matrices:

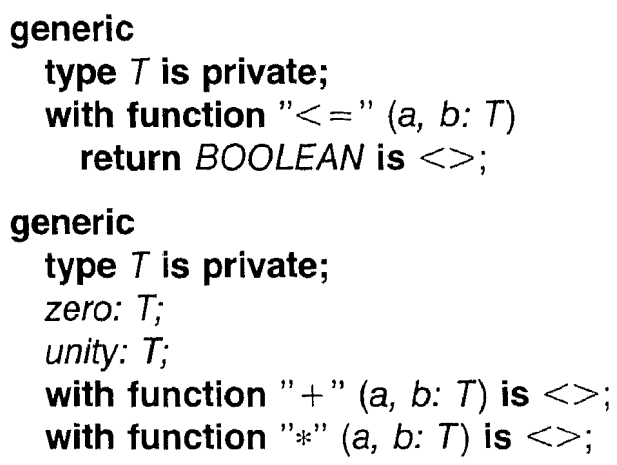

We may view these clauses as the definitions of two abstract data types say COMPARABLE and $R I N G$; the former is characterized by a comparison operation "<=", and the latter by features zero, unity, "+" and "*".

In an object-oriented language, such types may 


\section{Genericity versus Inheritance}

be directly represented as classes. We cannot define these classes entirely, for there is no universal implementation of " $<=$ ", " +", etc.; rather, they are to be used as ancestors of other classes, corresponding to actual generic parameters. The deferred feature mechanism of Eiffel provides exactly what is needed to express such classes

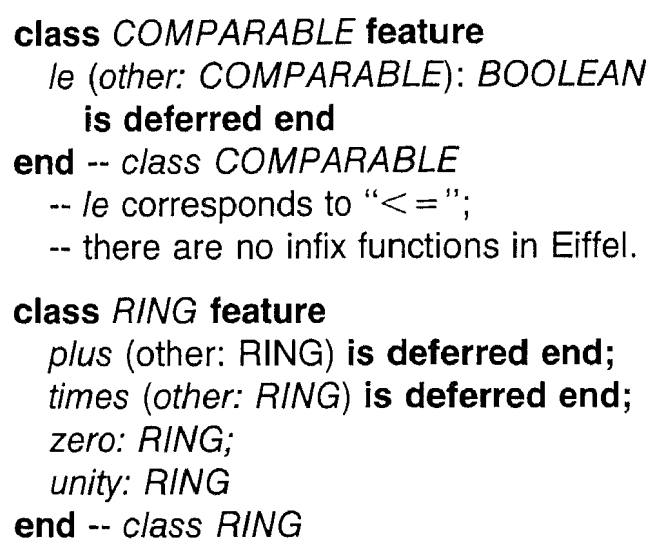

The comment made earlier about the lack of semantic specification in Ada constrained genericity would seem to apply here too: we have not specified any of the required properties on le, plus, etc. Eiffel does, however, permit the specification of such properties in the form of preconditions and postconditions on routines. Simple examples of this facility will be given later on.

The reader will also have noted that plus and times are defined here as procedures rather than functions; the convention we will follow in the Eiffel examples is that r.plus ( $\mathrm{r} 1$ ) is an instruction that performs a side-effect on $r$, adding to its value the value of $r 1$, rather than an expression returning the sum of these values (and similarly for times). In contrast, the Ada operators " + " and "*" were functions. The difference is not essential and we use procedures in Eiffel mainly for brevity. Subject to the following discussion, the examples may be changed into functions, as in

plus (other: RING): RING is deferred end;

\section{Constrained Genericity: Subprograms}

A subprogram such as minimum may now be written by specifying its arguments to be of type COMPARABLE. Based on the Ada pattern, the function would be declared as minimum (one: COMPARABLE; other:

COMPARABLE):

COMPARABLE is

-- Minimum of one and other

$$
\text { do ..... end }
$$

In an object-oriented language, however, every routine (Eiffel term for subprogram) appears in a class and is relative to the "current" object of that class; thus it seems preferable to include minimum in class COMPARABLE, argument one becoming the implicit current object. The class becomes

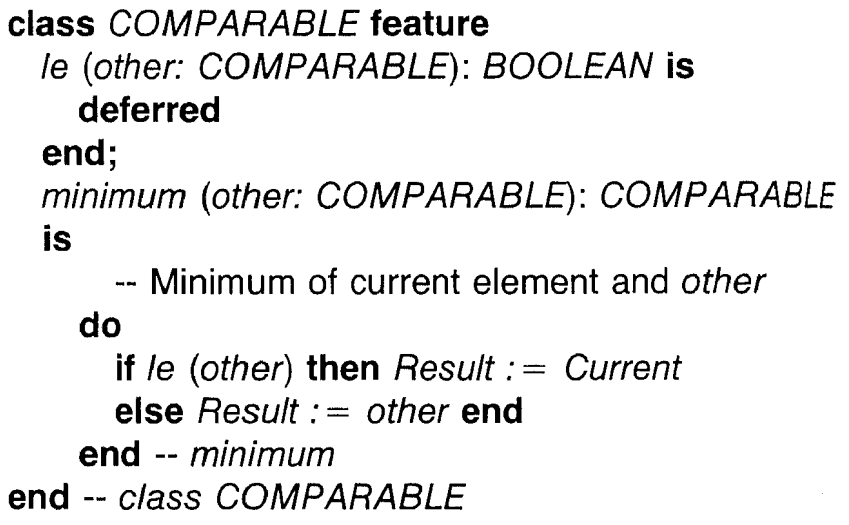

(The predefined variable Result contains the result to be returned by any function in which it appears; it is implicitly declared of the function's result type, here COMPARABLE.) To compute the minimum of two elements, we must declare them of some descendant type of COMPARABLE. For example, we may declare

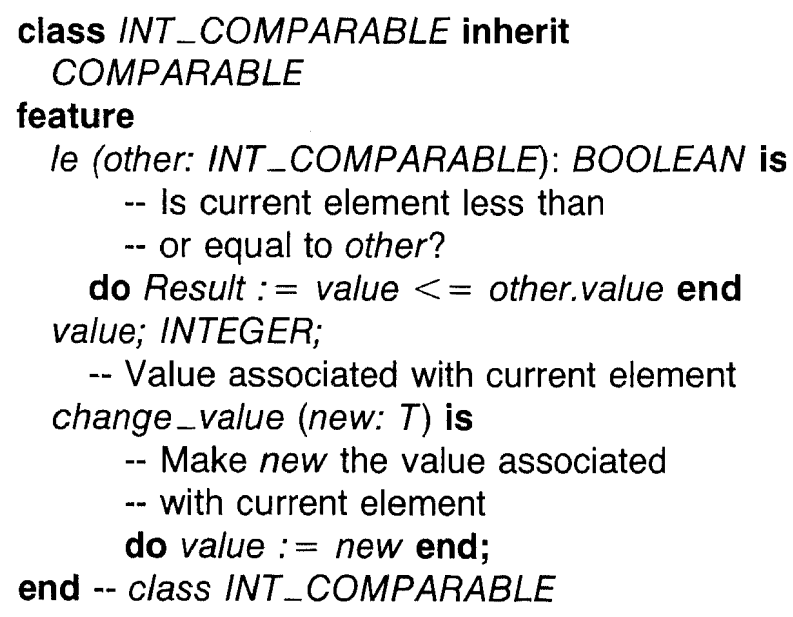

To find the minimum of two integers, we may now apply function minimum, not to arguments of type integer, but to arguments of type INT_COM$P A R A B L E$, say $i c 1$ and $i c 2$, as follows 


\section{Genericity versus Inheritance}

$i c 3:=$ ic1.minimum (ic2)

To use the generic le and minimum functions, we have to renounce direct references to integers, using INT_COMPARABLE entities instead; hence the need for attribute value and routine change value to access and modify the associated integer values.

We would similarly introduce heirs of $C O M$ $P A R A B L E$, say STR_COMPARABLE, REAL_ $C O M P A R A B L E$, and so on, for each type for which a version of minimum is desired.

Of course, having to declare similar features value and change_value for all descendants of COM$P A R A B L E$ is unpleasant. But by paying this relatively small price in terms of ease of program writing-renouncing the direct use of predefined typeswe seem to achieve the effect of genericity.

There is a hitch, however, if we are concerned about static typing. We clearly want to disallow a call such as

\section{ic1.minimum $(c)$}

where $c$ is a COMPARABLE but not an INT_COM$P A R A B L E$. Function le has indeed been redefined to accept only INT_COMPARABLE arguments; the rules of Eiffel permit such redefinition of an entity of a class in a descendant of that class, if the new type is itself, as here, a descendant of the original type. But minimum has not been redefined; in fact this is the whole point of the game: to make sure that minimum is a polymorphic feature, applicable to all kinds of "comparable" objects. So, regrettably, $c$ is in fact a legal argument as used above.

To ensure type consistency we must redefine minimum in INT_COMPARABLE so that its arguments and result are of type INT_COMPARA$B L E$. The body of the routine does not change: only its header has to be modified. The class declaration may thus be rewritten as follows

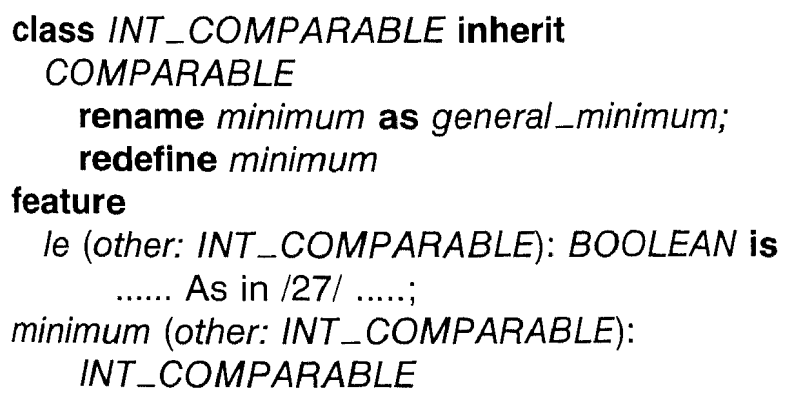

-- Minimum of current element and other

\section{do}

Result : = general_minimum (other)

end; -- minimum

value: INTEGER; -- As above

change_value (new: $T$ ) is -- As above

do value : = new end;

end -- class INT_COMPARABLE

We have used here the renaming mechanism of Eiffel; the rename... subclause of the inherit... clause makes it possible to access the features of the ancestor class (COMPARABLE) even though they are redefined in the descendant. Eiffel prohibits overloading of names within a class, so that renaming is necessary to allow use of both sets of features in the class. (Another use of renaming is in multiple inheritance, to remove name clashes when features are inherited from more than one class).

What we have done is to redefine the header of minimum-not its body, which remains the original one, made accessible under the name general minimum. This seems to take care of the static typing conflict, while introducing yet more complication.

However, the careful reader will have noted that a serious typing problem remains. The call to general_minimum is correct with respect to its argument other: since general_minimum (that is to say, COMPARABLE's version of minimum, as given on p. expects COMPARABLE objects, an entity like other declared of the descendant type INT_COMPARABLE is an acceptable substitute under the assignment rule. But there is a problem with the result of the function: general_minimum returns a $C O M$ PARABLE whereas INT_COMPARABLE's version of minimum should return an INT_COMPARABLE.

$i c 3:=$ ic1.minimum (ic2)

ic3 should be an INT_COMPARABLE; the assignment is illegal if the right-hand side returns just a COMPARABLE. In fact, the permitted type combinations in assignments are the inverse ones: the source should be of a descendant type from the target.

With what we have seen so far there is no way to resolve this issue other than by redefining minimum completely-not only its header, but its body as well - so that it will indeed return an INT_COM$P A R A B L E$. This of course defeats the whole purpose of genericity: a similar redefinition must be repeated in each descendant of COMPARABLE, with all instances of minimum identical except for the type declarations of arguments and results.

We shall only be able to provide a satisfactory 


\section{Genericity versus Inheritance}

solution to this problem by introducing declaration by association.

\section{Constrained Genericity: Packages}

The previous discussion transposes to packages. We use a class to represent the matrix abstraction implemented in Ada by the MATRICES package

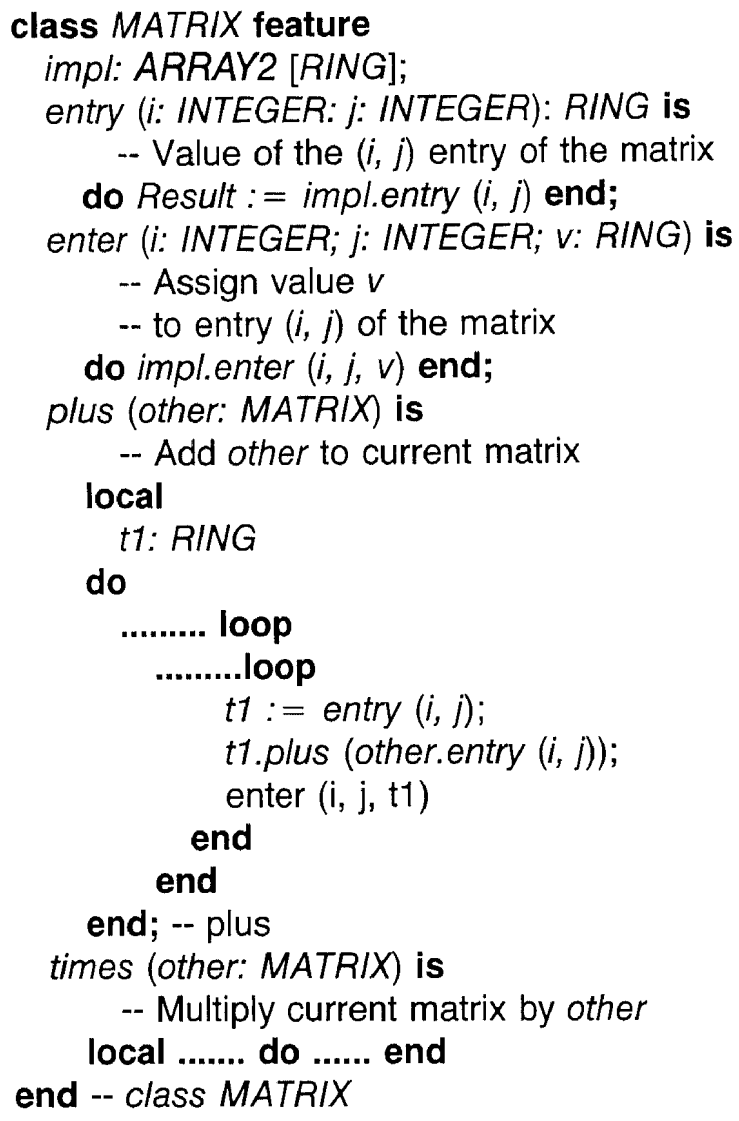

Here ARRAY2 [T] denotes a predefined Eiffel class whose elements are two-dimensional arrays of type $T$. Array types are treated in Eiffel as class types; the basic operations on an element $a$ of type ARRAY2 are a.entry $(i, j)$, which returns the $i, j$ entry of array $a$ (that is to say, $a[i, j]$ in standard Pascal notation), and a.enter ( $i, j, v)$, which assigns value $v$ to this entry (that is to say, $a[i, j]:=v$ ). Corresponding operations are declared above for matrices.

We have left out some details (such as how the dimensions of a matrix are set) but outlined the plus procedure, exhibiting the object-oriented form of overloading: the internal call to plus is the operation on RING, not MATRIX. Similarly, routines enter and entry are used in both their ARRAY2 and MATRIX versions.
To define the equivalent of the Ada generic package instantiation

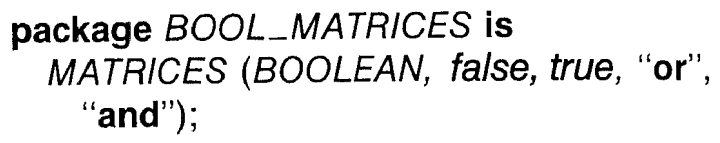

we must declare the "ring" corresponding to booleans

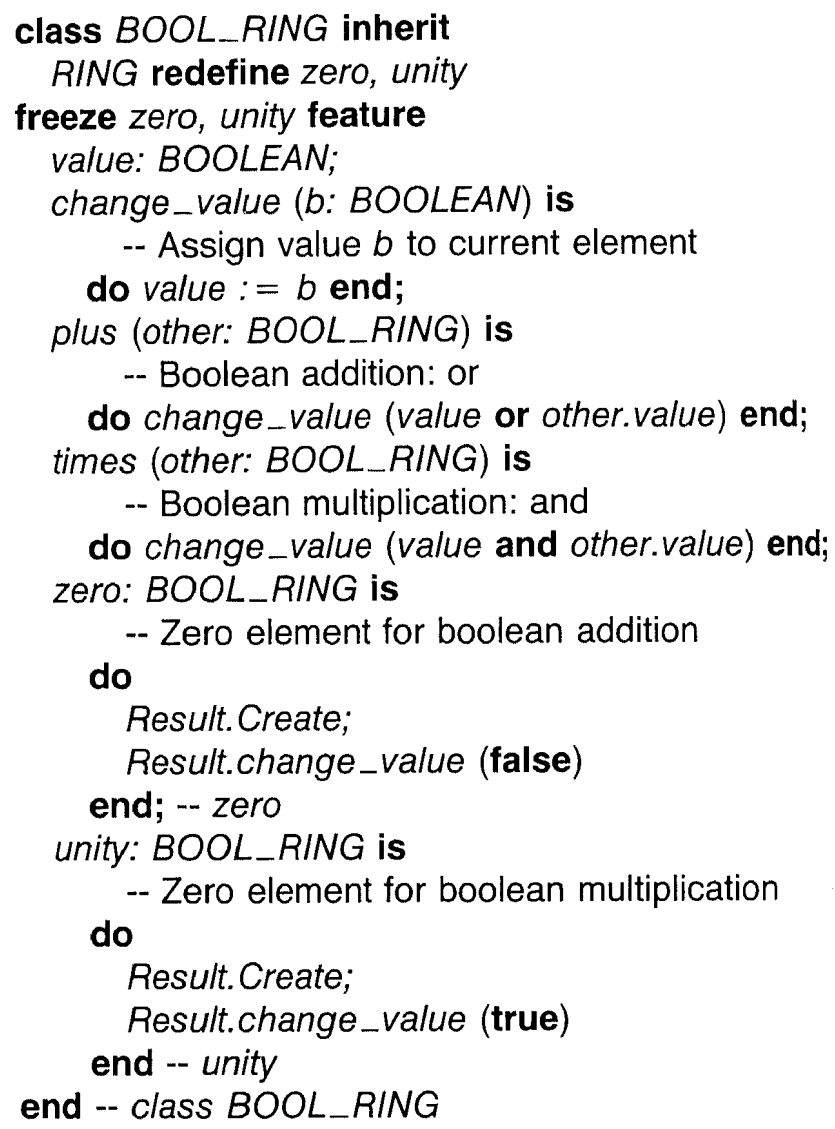

Note that zero and unity are redefined as functions returning a value of type $B O O L \_R I N G$. However, these are actually constant functions: the clause freeze ..., not seen before, indicates that zero and unity are evaluated just once and their values shared among all instances of the class. This is how constants of class types may be introduced in Eiffel.

How do we provide the equivalent to the Ada package instantiation for boolean matrices recalled above? The same reasoning that was applied to class COMPARABLE and function minimum prevents us from keeping MATRIX as it is if type checking is a concern: we want to make sure that an integer element, say, may not be entered into a boolean matrix. To achieve this, we define an heir BOOL_MATRIX of MATRIX, where routines entry, enter, plus and * are redefined to act only on objects of type $B O O L_{-}$ $R I N G$ rather than any RING. As with minimum, 


\section{Genericity versus Inheritance}

only the headers of the routines have to be changed, not their implementations; this is achieved as follows, using again renaming to allow access to redefined features of the parent class.

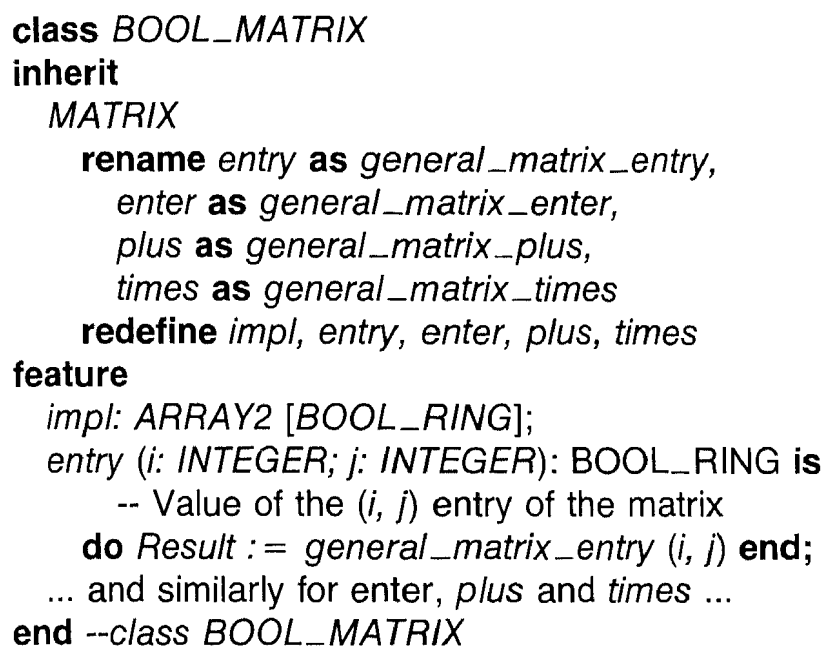

The reader may note the same problem for the result of function entry as previously discussed for minimum: this result should be of type $B O O L \_R I N G$, but general_matrix_entry will only return a RING. With the language features seen so far, all we can do is to redefine the body of entry, making it a copy of the body of general_matrix_entry rather than a call to this routine; then the result will be of the right type. Note that the problem only arises for functions, so the other routines of the class are not affected.

This problem notwithstanding, the construction achieves with inheritance the effect of constrained genericity. The price to pay is a certain heaviness in expression; note in particular that what has been done for $B O O L \_M A T R I X$ must be repeated for any descendant of MATRIX representing a generic instantiation, as INT_MATRIX, REAL_MATRIX, etc. Furthermore, features value and change -value must be declared anew in each descendant of the associated class RING. We shall see later how such heaviness may be removed.

\section{Unconstrained Genericity}

The mechanism for simulating unconstrained genericity is the same; this case is simply seen as a special form of constrained genericity, with an empty set of constaints. As above, formal type parameters will be interpreted as abstract data types, but here with no relevant operations. The technique works, but suffers from the heaviness mentioned above, be- coming less tolerable here as the dummy types do not correspond to any obviously relevant data abstraction.

Let us apply the previous technique to both our unconstrained examples, swap and stack, beginning with the latter. We need a class, say $S T A C K$ $A B L E$, describing objects that may be pushed onto and retrieved from a stack. Since this is true of any object, this class has no property beyond its name:

\section{class STACKABLE end}

We may now declare a class $S T A C K$, whose operations apply to $S T A C K A B L E$ objects

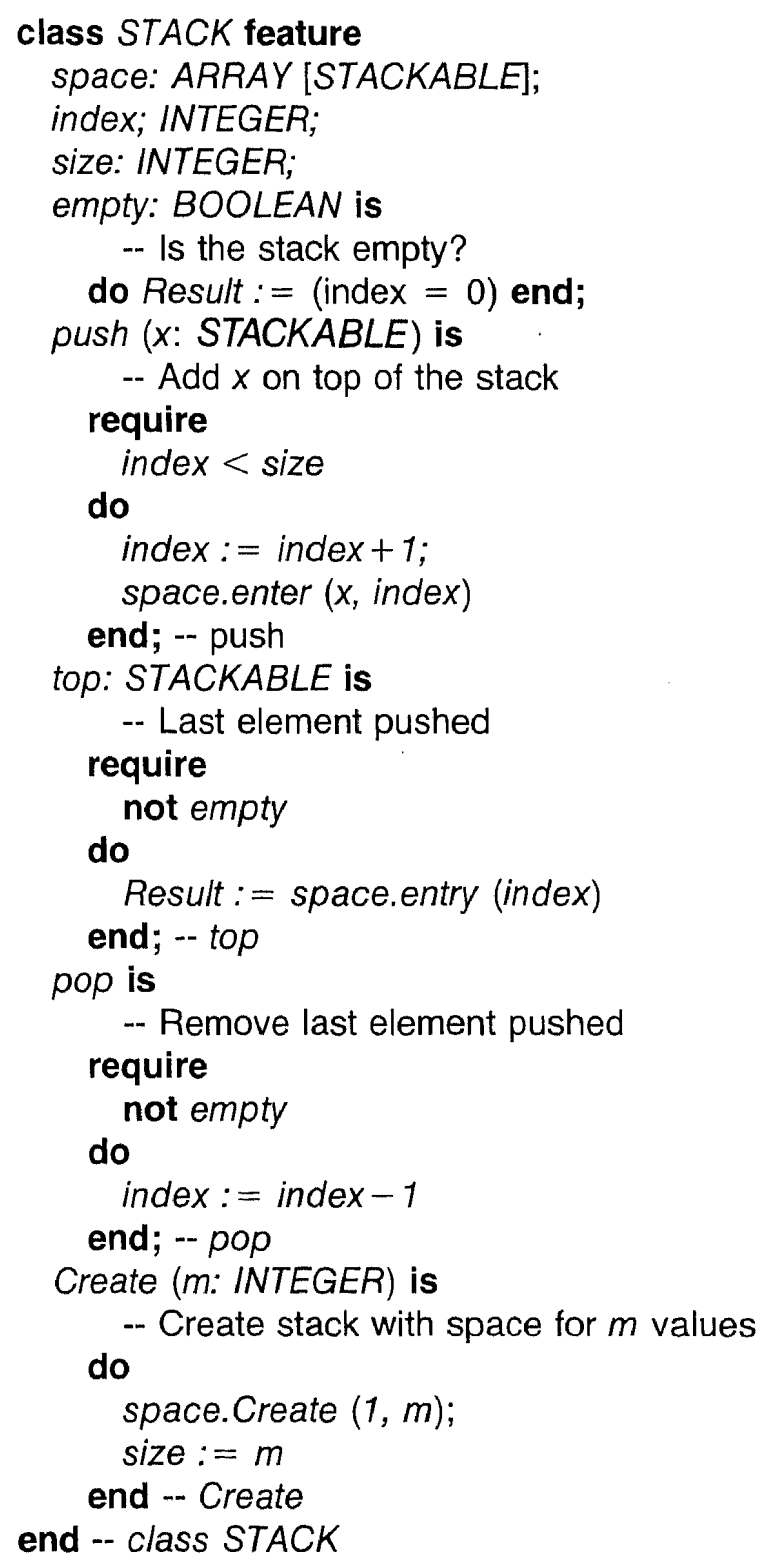

The require... clauses illustrate how routine preconditions (which must be satisfied by actual pa- 


\section{Genericity versus Inheritance}

rameters upon entry to a routine) are written in Eiffel. Postconditions and class invariants may also be expressed (in ensure... and invariant... clauses). This aspect of the language falls beyond the scope of this discussion; see Meyer [12] for more details.

$S T A C K$ relies on the predefined class ARRAY for one-dimensional arrays, whose main procedures are entry, enter, and Create; the latter takes two arguments and allocates the array with the values of these arguments as bounds. The Create procedure for stacks takes just one argument (the stack size).

To instantiate this definition for stacks of specific types, we apply the same techniques as above: define descendants of STACKABLE, such as

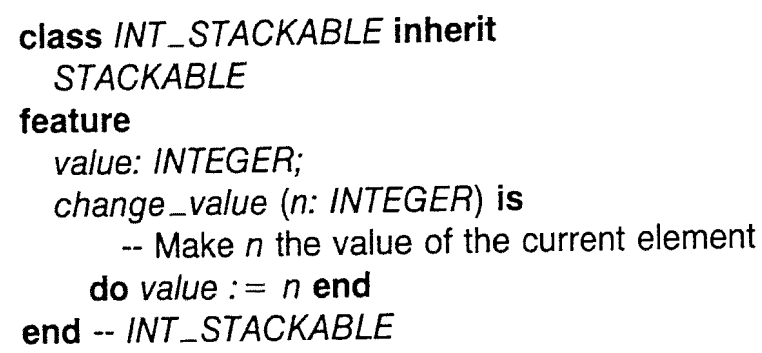

and similarly STR_STACKABLE, etc.

Here we run again into the typing problem evidenced by minimum and BOOL_MATRIX. Stacks declared simply of type STACK cannot be statically guaranteed to contain only objects of a certain class of "stackables," say INT_STACKABLE; and we have the problem of the type of the result returned by function top. In the following sequence

\section{s: STACK; ins: INT_STACKABLE}

s.Create (10);

ins.Create; ins.change_value (50);

s.push (ins);

ins : $=$ s.top

the last assignment has a left-hand side of type INT_STACKABLE and a right-hand side of type $S T A C K A B L E$; this is typewise wrong even though the code seems quite legitimate semantically (one pushes the value of a variable and retrieves it immediately into the same variable).

For both these reasons, it is necessary to do as in the previous examples, that is to say declare heirs to STACK, such as INT_STACK, STR_STACK etc. Features of STACK will be redefined in each of these classes, but only to adapt the types of their arguments and, in the case of top, of the result. Thus for example, INT_STACK will contain feature redefinitions such as space: ARRAY [INT_STACKABLE];

push ( $(x:$ INT_STACKABLE) is

do general_stack_push $(x)$ end;

etc. (the reader may complete this example based on the MATRIX case).

The other unconstrained example, procedure swap, may be treated along the same lines; a class $S W A P P A B L E$ will be introduced. The treatment is left to the reader.

\section{GENERICITY AND INHERITANCE IN EIFFEL}

We may draw the following conclusions from the previous discussion.

- Inheritance is the more powerful mechanism. There is no way to provide a reasonable simulation with genericity.

- The equivalent of generic subprograms or pack. ages may be expressed in a language with in. heritance, but one does not avoid the need for certain spurious duplications of code. The extra verbosity is particularly hard to justify in the case of unconstrained genericity, for which the simulation mechanism is just as complex as for the conceptually more difficult constrained case.

- Type checking introduces difficulties in the use of inheritance to express generic objects.

To address these issues, Eiffel offers a limited form of genericity and the notion of declaration by association. (The specification language LM, associated with the $M$ specification method [15], relies on a similar tradeoff.)

\section{Simple Genericity}

Since unconstrained genericity is both the simpler case and the one for which the pure inheritance solution is least acceptable, it seems adequate to provide a specific mechanism for this case, distinct from the inheritance mechanism. Consequently, Eiffel classes may have unconstrained generic param. eters. A class may be defined as

class $C[T 1, T 2, \ldots, T n] \ldots$.

where the parameters represent arbitrary types (simple or class). An actual use of the class will use actual type parameters, as in

$x:$ C [INTEGER, RING, ..., DEVICE] 


\section{Genericity versus Inheritance}

We have in fact already encountered such parameterized classes: the basic classes $A R R A Y$ and $A R R A Y 2$ are naturally generic. It should also be noted (although the present paper is about concepts rather than implementation) that Eiffel compilation techniques make it possible to generate a single object module for a parameterized class, as opposed to Ada techniques which treat generic packages as macros to be expanded anew for each instantiation.

The examples of the previous sections provide obvious cases where generic parameters are useful. For instance COMPARABLE becomes

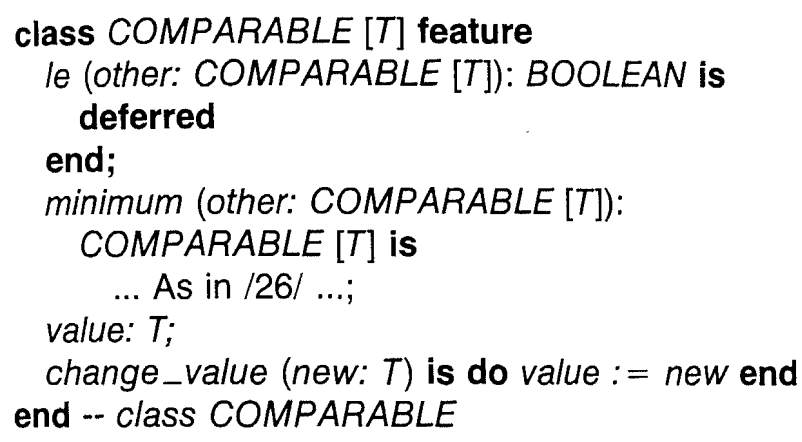

Here we see an immediate and important benefit of generic parameters: we can solve almost completely the problem of type checking by specifying that the arguments to le and minimum and the local variable $m$ are of type COMPARABLE [T], for the same $T$ as the class itself. Thus we rid ourselves of the necessity to redefine, at least formally, minimum for each descendant of COMPARABLE, which plagued our previous attempts. The generic parameter $T$ also allows us to lift the declarations of features value and change_value from the various descendants of COMPARABLE to a single instance in COMPA$R A B L E$ itself.

However, we have not yet found a proper type for minimum's result, which remains a COMPA$R A B L E[T]$ even in descendants; more on this below.

To define INT_COMPARABLE all we have to write now is

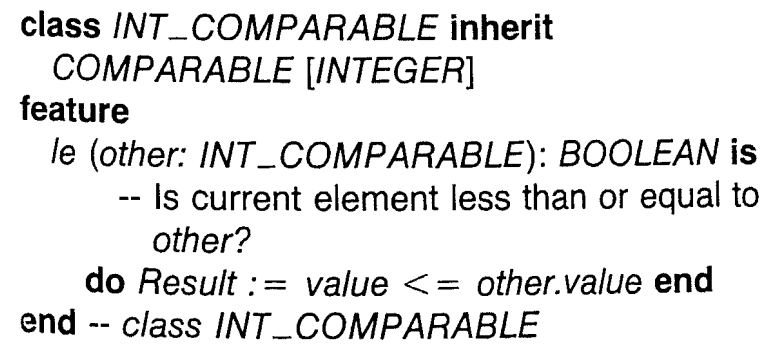

The other examples are treated similarly

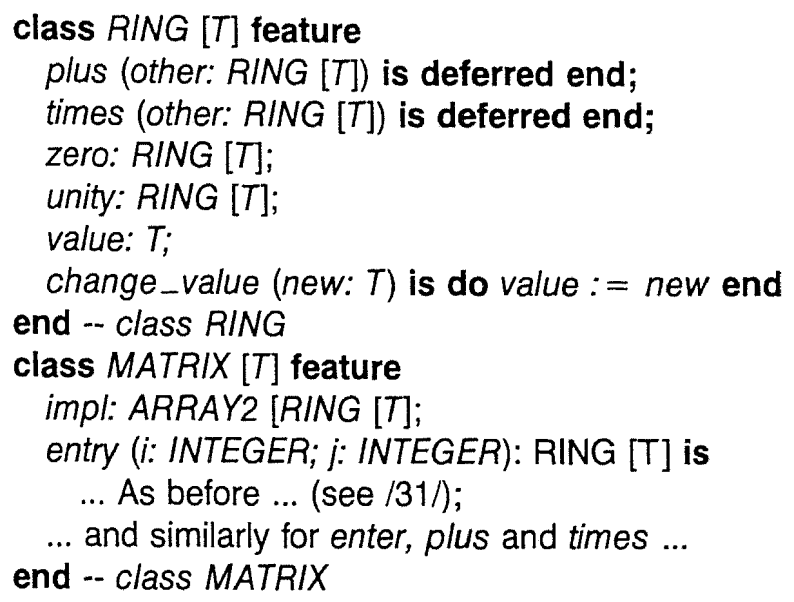

Note how the use of a generic parameter in two related classes, RING and MATRIX, makes it possible to ensure type consistency (all elements of a matrix will be of type RING [T] for the same $T$ ). As with COMPARABLE, the declarations of features value and change_value have been factored out: they now appear in class MATRIX rather than being repeated in all its descendants.

In the unconstrained case, the need for dummy classes disappears; class STACKABLE and its heirs INT_STACKABLE, STR_STACKABLE, etc. are not needed any more, since STACK may be rewritten as

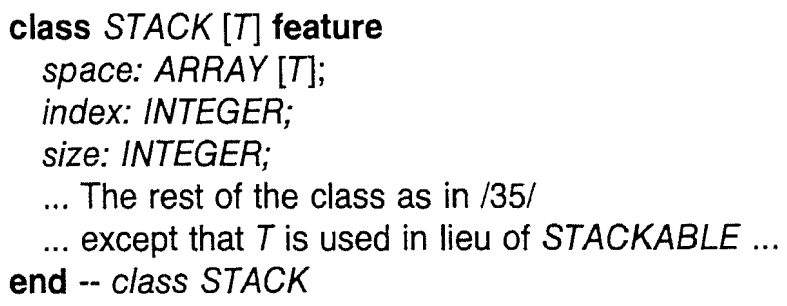

There is also no more need for classes such as INT_STACK, STRING_STACK, etc.; simply use STACK [INTEGER], STACK [STRING], and so on. The typing problem for top disappears since the result of this function is now simply of type $T$.

A remarkable degree of simplification has been achieved. Auxiliary classes are not needed any more for unconstrained genericity. However, we do not introduce constrained genericity in the language: this feature would be redundant with the inheritance mechanism. To provide the equivalent of a constrained formal generic parameter, we retain the technique introduced earlier: declare a special class whose features correspond to the constraints (that is to say, the with subprograms in Ada terminology), and declare any corresponding actual parameters as descendants of this class. Providing the class with 


\section{Genericity versus Inheritance}

generic parameters simplifies its use and partly solves the type checking problem.

\section{Declaration by Association}

Let us look more closely at the remaining part of the type checking problem. Consider again class COMPARABLE as defined last. Keeping in mind that COMPARABLE is intended for use as an ancestor for more specific classes, we do not really want other (in both functions), $m$ and the result of minimum to be of type COMPARABLE [T]: what is required of these entities is to be of the type of the "current" entity, whatever this may be in a descendant of COMPARABLE. When this type changes, we want the other entities to follow suit.

This possibility is achieved in Eiffel through the mechanism of declaration by association. Let a class $C$ contain a declaration of the form

$x: D$

where $D$ is a class type. We may then declare another entity as

$y$ : like $x$

Such a declaration means the following: the type of $y$ is the same as the type of $x$; if $x$ is redefined in a descendant class of $C$ as being of a class type $D^{\prime}$, which must be a descendant of $D$, then $y$ will be considered to have been redefined likewise. Note that this is a purely static mechanism; it may be viewed as an abbreviation allowing the redeclaration of just one from a group of related entities to stand for the redeclaration of the whole group.

When this distinguished entity, $x$ above, is redeclared, it "drags" along all entities declared like it. We call it the anchor of the association. The anchor may be the current entity, as in

\section{y: like Current}

This readily applies to the previous example

class COMPARABLE [T] feature -- Contrast with 139/

le (other: like Current): BOOLEAN is deferred

end;

minimum (other: like Current): like Current is do ... see /26/ ... end; value: $T$;

change_value (new: $T$ ) is do value := new end end -- class COMPARABLE

Note how this device solves at once all the remaining type checking problems: not only are le and minimum constrained to act, in all descendants of COMPARABLE, on homogeneous entities (com. paring only integers with integers, strings with strings, etc.); it also ensures that the result of min. imum is of the right type, that of its arguments.

The same technique readily applies to the other cases. For example, RING becomes

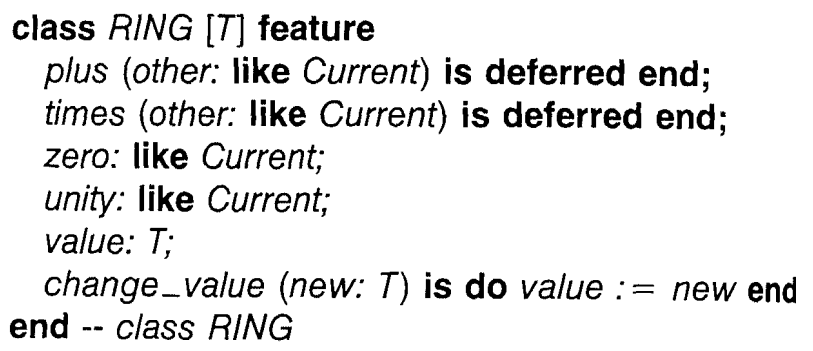

In contrast with the STACK case, we do need here, because of the deferred procedures, to explicitly declare descendants of RING for various implemen. tations of plus and times; for example

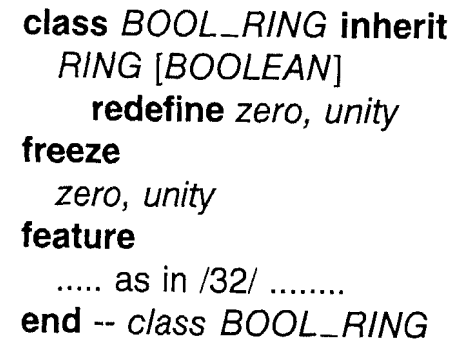

\section{Artificial Anchors}

For MATRIX, a small addition is necessary to ensure that all entities of type RING $[T]$ are always redefined consistently.

When a group of entities are redefined together by association, one of the entities must serve as the anchor for the association. In the final versions obtained above for COMPARABLE and RING, the current element is the anchor.

In MATRIX, the entities to be redefined are of a type, namely RING, different from the current class. In such a case, the class usually contains a feature of the required type which can serve as anchor. For example, the basic Eiffel library [12] in- 


\section{Genericity versus Inheritance}

cludes an implementation of linked lists through two classes: LINKED_LIST [T] for the lists themselves and LINKABLE [T] for list cells (a cell contains a value of type $T$ and a reference to another cell). The implementation of a list contains a reference first element to the first cell of the list; first_element is used as anchor for other LINKABLE entities of class LINKED_LIST and redefined in descendants of LINKED_LIST, such as the classes for two-way lists and trees (both viewed as special cases of linked lists).

Class MATRIX, however, has no feature of type $R I N G$; the reason is that "ring" elements are entered into the matrix indirectly, as arguments to procedure entry. Thus, we cannot avoid the need for a dummy feature of type RING serving as anchor:

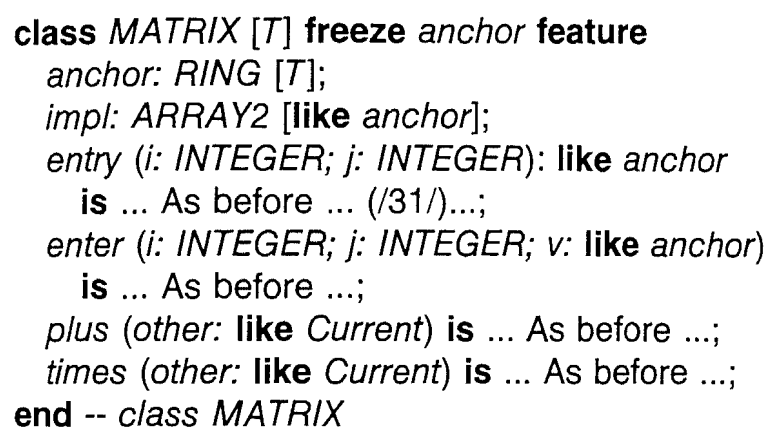

(Listing anchor in the freeze clause avoids the waste of run-time space that would result from physically storing an anchor within each object of the class.) Here too specialized classes must be declared for various generic instances of MATRIX. However, the declarations are now trivial: all that needs to be done is to redefine anchor. For example

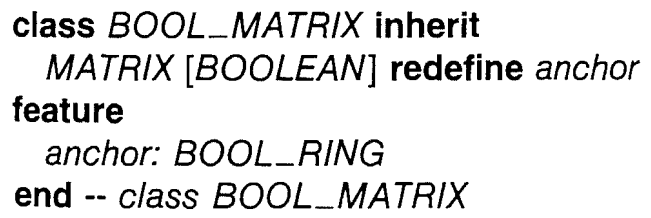

Such a redeclaration closely models the corresponding Ada package instantiation (/12/).

\section{CONCLUSION}

Genericity and inheritance are two important techniques towards the software quality goals mentioned at the beginning of this article. We have tried to show which of their features are equivalent, and which are complementary.

Providing a programming language with the full extent of both inheritance and Ada-like genericity would, as we think this discussion has shown, result in a redundant and overly complex design; but including only inheritance would make it too diffcult for programmers to handle the simple cases for which unconstrained genericity offers an elegant expression mechanism, like in the stack example.

Thus we have put the borderline at unconstrained genericity. Eiffel classes may have unconstrained generic parameters; constrained generic parameters are treated through inheritance.

Declaration by association completes this architecture by allowing for completely static type checking, while retaining the necessary flexibility.

We hope to have achieved in this design a good balance between the facilities offered by two important but very different techniques for the implementation of extendible, compatible, and reusable software.

\section{Acknowledgments}

This work benefited from comments by Vincent Cazala and was done in part as the author was with the University of California, Santa Barbara.

\section{References}

1. Jean-Raymond Abrial, Stephen A. Schuman, and Bertrand Meyer, "A Specification Language," in On the Construction of Programs, ed. R. McNaughten and R.C. McKeag, Cambridge University Press, 1980.

2. Didier Bert, "Manuel de Référence du Langage LPG, Version 1.2," Rapport R-408, IFIAG, IMAG Institute (Grenoble University), Grenoble, December 1983.

3. Graham Birtwistle, Ole-Johan Dahl, Bjorn Myrhaug, and Kristen Nygaard, Simula Begin, Studentliteratur and Auerbach Publishers, 1973.

4. Ronald J. Brachman, "What IS-A and isn't: An Analysis of Taxonomic Links in Semantic Networks," Computer (IEEE), vol. 16, no. 10, pp. 67-73, October 1983.

5. Rod M. Burstall and Joe A. Goguen, "An Informal Introduction to Specifications using Clear," in The Correctness Problem in Computer Science, ed. R.S. Boyer and J.S. Moore, pp. 185-213, Springer-Verlag, New York, 1981.

6. Luca Cardelli and Peter Wegner, "On understanding Types, Data Abstraction and Polymorphism," Computing Surveys (to appear).

7. Luca Cardelli, "Basic Polymorphic Typechecking," AT\&T Bell Laboratories Computing Science Technical Report, 1984, 1986. (Revised version, to appear).

8. Ole-Johan Dahl, Bjørn Myrhaug, and Kristen Ny- 


\section{Genericity versus Inheritance}

gaard (Simula) Common Base Language, Norsk Regnesentral (Norwegian Computing Center), Oslo, February 1984.

9. K. Futatsugi, Joseph A. Goguen, Jean-Pierre Jouannaud, and José Messeguer, "Principles of OBJ2," in Proceedings of the 1985 ACM Symposium on Principles of Programming Languages, vol. 12, pp. 52-66, 1985.

10. Barbara H. Liskov, R. Atkinson, T. Bloom, E. Moss, J.C. Schaffert, R. Scheifler, and Alan Snyder, CLU Reference Manual, Springer-Verlag, Berlin-New York, 1981.

11. Bertrand Meyer, "Quelques concepts importants des langages de programmation modernes et leur expression en Simula 67," Bulletin de la Direction des Etudes et Recherches d'Electricité de France, Série C (Informatique), no. 1, pp. 89-150, Clamart (France), 1979. Also in GROPLAN 9, AFCET, 1979.

12. Bertrand Meyer, Eiffel: a Language for Software Engineering, Technical Report TRCS86-4, Interactive
Software Engineering, Version 2.1, August 1986, №. vember 1985 (Revised, August 1986).

13. Bertrand Meyer, "Eiffel: Programming for Reusability and Extendibility," ACM Sigplan Notices, 1987. (To appear)

14. Bertrand Meyer, Object-oriented Software Construc. tion, 1987. (To appear)

15. Bertrand Meyer, "M: A System Description Method," Technical Report TRCS85-15, University of Califor. nia, Santa Barbara, Computer Science Department, May 1985.

16. Robin Milner, "A Theory of Type Polymorphism in Programming," Journal of Computer and System Sciences, vol. 17, pp. 348-375, 1978.

17. Rishiyur S. Nikhil, "Practical Polymorphism," in Functional Programming Languages and Computer Architecture, Nancy (France), 16-19 September 1985, Lecture Notes in Computer Science 201, ed. Jean-Pierre Jouannaud, pp. 319-333, Springer-Verlag, Berlin-New York, 1985. 